\title{
A METHOD FOR RECEIVED WAVEFORM RECONSTRUCTION BASED ON BENDER ELEMENT TEST USING FREQUENCY-SWEPT SIGNAL
}

\author{
Toshiniro OGino ${ }^{\text {i) }}$, ToshiYuki Mitachii ${ }^{\text {ii }}$, KoK Hoor Chan ${ }^{\text {ii) }}$, \\ Hiroshi Oikawa ${ }^{\text {iii) }}$ and Masaki Tsushima ${ }^{\text {iv) }}$
}

\begin{abstract}
An experimental technique for reconstructing the received wave of bender element tests based on linear system theory is shown in this paper. In order to identify the frequency response of testing apparatus, bender element tests using frequency-swept signals are performed. Received waves for one-period sine pulse transmitting with various frequencies are calculated and compared with observed waves in several kinds of soil samples and testing apparatuses. The linearity of the testing system is also confirmed by coherence function. It is shown that calculated data are less affected by random noise and show good agreement with observed data in regards to not only waveform itself but also resulting shear wave velocity. It is mentioned that this technique can provide simulations for arbitrary transmitted waveform with high signal/noise ratio after laboratory tests are performed. It is also mentioned that this technique is advantageous when sufficient amplitude of received wave cannot be ensured or shear wave velocity must be verified, for example, due to uncertainty of arrival time by near-field effect.
\end{abstract}

Key words: (bender element), laboratory test, peat, (shear wave velocity), test procedure (IGC: D7)

\section{INTRODUCTION}

After being introduced by Shirley and Hampton (1978) for soil testing, bender elements have been installed by many researchers in various test apparatuses such as oedometer, triaxial or simple shear apparatus etc. in order to measure shear wave velocity in the laboratory (Dyvik and Madshus, 1985; Dyvik and Olsen, 1991; Brignoli et al., 1996; Viggiani and Atkinson, 1995). They determined shear wave velocity $V_{\mathrm{s}}$ by the following equation through travel time measurement.

$$
V_{\mathrm{s}}=\frac{L}{t}
$$

where $L$ is the tip-to-tip distance between transmitter and receiver element and $t$ is travel time defined as the distance between start and arrival point from time trace of oscilloscope. However, it is pointed out as an important issue that distortion of received waveform often masks the arrival point. Sanchez-Salinero et al. (1986) numerically showed how near-field effect affects the received waveform in relation to $\lambda$ and $L$ (where $\lambda$ is wavelength). Brignoli et al. (1996) also experimentally showed that near-field components may complicate the arrival time detection based on simultaneous measurement of compression and shear wave. Arulnathan et al. (1998) men- tioned that observed received wave could be affected by reflected wave at rigid boundary. Due to these difficulties, frequencies and waveforms of transmitter signal have not been prescribed yet as well as method of travel time estimation has not been standardized. As a result, some researchers defined arrival time at first deflection point of received wave using one-period sine pulse, while others defined at zero-crossing point using square wave.

Alternatively,other interpretations to determine the travel time were suggested. Viggiani and Atkinson (1995) showed a method based on cross-correlation analysis. Arulnathan et al. (1998) used cross-spectrum of transmitted and received wave. Greening and Nash (2004) determined travel times corresponding to phase and group velocities using phase shift of each frequency component comprised in received wave for frequency-swept signal excitation.

Conditions to avoid occurrence of near-field effect have been also shown so far (Sanchez-salinero et al., 1986; Jovicic et al., 1996). From an analytical solution Jovicic et al. (1996) concluded that near-field effect disappeared when $L / \lambda$ became large. Since wavelength decreases by increasing frequency of transmitter signal, higher frequency can avoid the occurrence of near-field effect. Researchers, however, proposed different value about the occurring condition of near-field effect. For example,

i) Akita University, Japan (ogino@ce.akita-u.ac.jp).

Hokkaido University, Japan.

Akita University, Japan.

iv) Akita National College of Technology, Japan.

The manuscript for this paper was received for review on September 18, 2007; approved on December 31, 2007.

Written discussions on this paper should be submitted before November 1, 2008 to the Japanese Geotechnical Society, 4-38-2, Sengoku, Bunkyo-ku, Tokyo 112-0011, Japan. Upon request the closing date may be extended one month. 
Sanchez-salinero et al. (1986) suggested $2 \leq L / \lambda \leq 4$, whereas Viggiani and Atkinson (1995) showed that nearfield effect occurred when $L / \lambda$ is approximately 2 or 3 . Moreover, wavelength cannot be known during testing because wavelength is not measureable but calculated from frequency and shear wave velocity at given travel time and distance. This means that frequency which will meet above conditions is also unknown at the time of testing. Thus it is still difficult for experimenters which point should be taken as arrival time or which frequency or waveform should be used for transmitter signal. For estimating reasonable arrival time, a practical method in which numbers of received waves for different transmitter waveforms are measured and recorded beforehand is often taken so that arrival time can be verified after testing.

On the other hand, Santamarina and Fam (1997) and Blewett et al. (2000) mentioned linearity of testing apparatus. Santamarina and Fam (1997) pointed out that frequency response of the system could be identified by impulse, white noise or frequency sweep excitation. This means that received wave for any transmitted signal can be obtained by convolution of impulse response with transmitted signal in time domain or product of frequency response and transmitted signal in frequency domain. The objective of this paper is to resolve the issue of difficulties in choice of transmitter signal by using this characteristic. An experimental technique to identify frequency response using frequency-swept signal, calculation of received wave for various frequency and comparison with observed waveform are described in this paper. The received wave reconstruction makes the verification of arrival time mentioned above more assured. The advantage of using frequency-swept signal in addition to using signals which have been used so far is also suggested.

\section{BENDER ELEMENT TESTING SYSTEM}

Bender element testing system can be interpreted as a linear system of which transmitted and received wave are input and output, respectively. Blewett et al. (2000) and Lee and Santamarina (2005) described that bender element testing system is composed of linear subsystems each of which should independently be determined by characteristics of transmitter and receiver bender elements and soil specimen. Santamarina and Fam (1997) mentioned that output of the system is represented by a damped, single-degree-of-freedom system subjected to forced vibration. Blewett et al. (2000) also suggested that the system is comprised of transmitter and receiver bender elements of which response is following harmonic oscillation and soil sample of which response is in a manner of Biot medium. Hence, output of the system should be a function governed by input and dynamic property of the system. Assuming linearity of the system, the output of the system for arbitrary input is represented by the convolution integral given by Eq. (2).

$$
y(t)=\int_{0}^{t} x(\tau) z(t-\tau) d \tau
$$

where, $y(t)$ and $x(t)$ are output and input, respectively, $t$ is time, $\tau$ is a parameter and $z(t)$ is impulse response of the system, which is equivalent to output for an impulse input. In frequency domain, Eq. (2) which is represented in time domain can be represented in a more simple expression given by Eq. (3).

$$
Y(f)=X(f) \cdot Z(f)
$$

where, $Y(f)$ and $X(f)$ are Fourier transform of $y(t)$ and $x(t)$, respectively and $Z(f)$ is frequency response function, which is equivalent to Fourier transform of impulse response $z(t)$. In Eqs. (2) and (3), $z(t)$ or $Z(f)$ comprises dynamic property of the testing system and should be affected by dimensions of transmitter and receiver benders and condition of specimen such as type of soil sample, dimensions, boundary condition, consolidation stress, and so on. Therefore, when $z(t)$ or $Z(f)$ is known, output of the system can be calculated analytically by a simple equation in frequency domain.

\section{FREQUENCY-SWEPT SIGNAL AND IDENTIFICATION OF FREQUENCY RESPONSE FUNCTION}

As mentioned by Santamarina and Fam (1997), there are some procedures for identifying frequency response function or impulse response of linear system. Frequency-swept signal is one of practical input signal to identify the frequency response function or impulse response. Since the meanings of impulse response is output of the system for unit impulse input, to use unit impulse as transmitter pulse may be considered as the simplest way. However, this method generally does not provide enough accuracy because of technical problems. Frequency-swept signal, often called chirp signal, is a signal of which frequency is stretched along time axis. This signal is equivalent to impulse in terms of the amplitude of each frequency component. This signal has an advantage in signal/noise ratio over other signals such as impulse or white noise.

In this study, two types of frequency-swept signal are used. First, Time-stretched pulse (TSP) is adopted. TSP, which is suggested by Aoshima (1981), is described by the following equation:

$$
X(k)=\left\{\begin{array}{l}
\exp \left(i \frac{m \pi k^{2}}{N^{2}}\right) 0 \leq k \leq \frac{N}{2} \\
X^{*}(N-k) \frac{N}{2}+1 \leq k \leq N
\end{array}\right.
$$

where $k$ is discrete frequency, $X(k)$ is Fourier transform of TSP, $m$ and $N$ are integer numbers and asterisk represents complex conjugate. $m$ relates to degree of frequency sweep: the larger value provides more stretched pulse in time domain. Secondly, a linearly swept sine pulse (LSSP) is adopted. LSSP is given by 


\section{Time Domain}
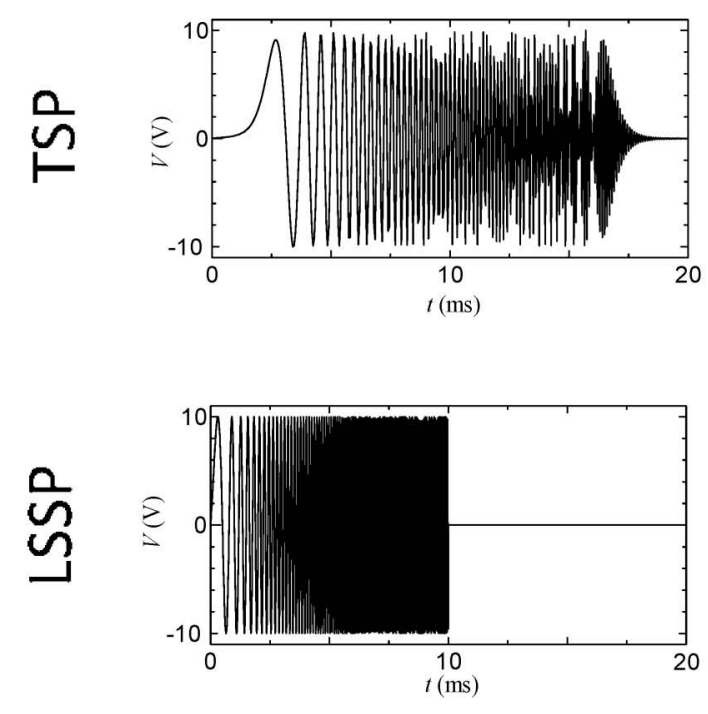

\section{Frequency Domain}
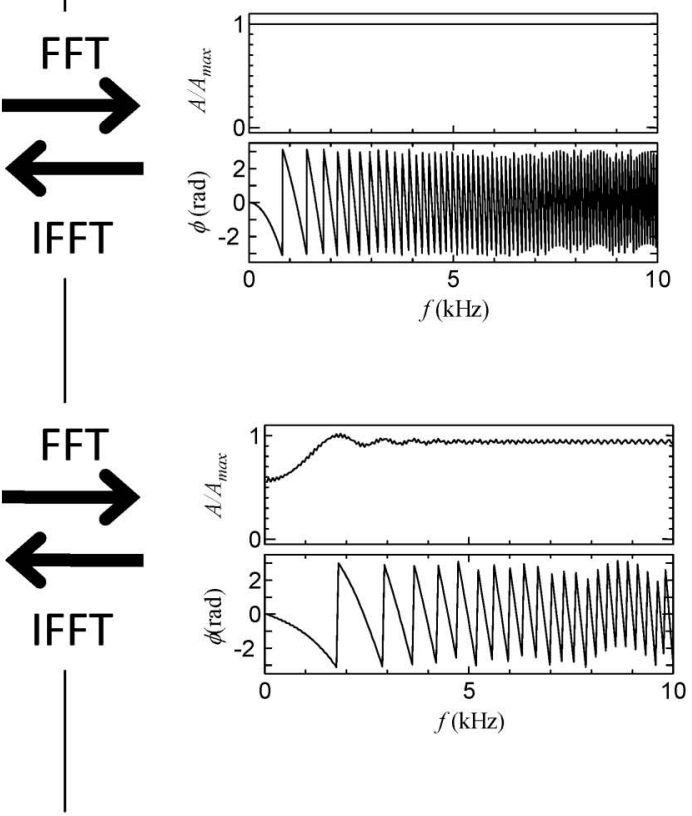

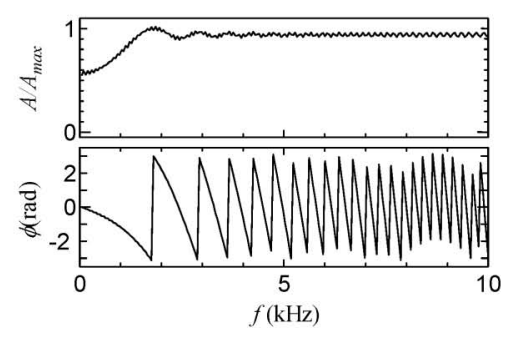

Fig. 1. TSP and LSSP in time and frequency domain given by Eqs. (4) and (5) (TSP: $m=150, N=2048$, LSSP: $f_{0}=0.5 \mathrm{kHz}, \Delta f=10 \mathrm{kHz}$ and $t_{\mathrm{t}}=$ $10 \mathrm{~ms})$

$$
\begin{aligned}
& x(t)=A \sin \omega t \\
& \omega=2 \pi\left(f_{0}+\frac{t}{t_{\mathrm{t}}} \Delta f\right)
\end{aligned}
$$

where $A$ is amplitude, $\omega$ is angular frequency, $t$ is time, $f_{0}$ is initial frequency, $\Delta f$ is bandwidth and $t_{\mathrm{t}}$ is target time. TSP where $m=150$ and $N=2048$ and LSSP where $f_{0}=0.5$ $\mathrm{kHz}, \Delta f=10 \mathrm{kHz}$ and $t_{\mathrm{t}}=10 \mathrm{~ms}$ are compared in both time and frequency domain in Fig. 1. Time histories of waveforms are shown in the left side of the figure in time domain and amplitude spectrum normalized by the maximum value $A_{\max }$ and phase spectrum are shown in the right side in frequency domain. Although these two signals are essentially the same in frequency-swept terms, it is found that there are differences in waveforms or amplitude and phase of each frequency component, that is, in LSSP, frequency is linearly shifted in proportion to time, whereas phase is shifted in proportion to square of frequency in TSP.

Inverse filter of frequency-swept signal is needed for reconstruction of frequency response. From its nature, inverse filter, which is given by complex conjugate of the signal, restores frequency-swept signal into an impulse. It follows that when received signal is put through the inverse filter, a received signal corresponding to an impulse is restored. Since applying inverse filter to signals can be represented by complex product in frequency domain, frequency response function is given by the following equation.

$$
Z(f)=X^{*}(f) \cdot Y(f)
$$

where $X(f)$ is Fourier transform of frequency-swept signal and $Y(f)$ is corresponding received signal in this equation. Note that Eq. (6) resulted in the cross-spectrum of $X(f)$ and $Y(f)$.

\section{LABORATORY EQUIPMENTS AND EXPERIMENTAL PROCEDURES}

Three kinds of samples: NSF clay, Kasaoka clay and Akita peat are used for the tests. NSF clay and Kasaoka clay are commercially available in a powder state. Particle density, liquid limit, plasticity index and clay content of each samples are $2.78 \mathrm{~g} / \mathrm{cm}^{3}, 55 \%, 26$ and $99 \%$ for NSF clay, and $2.77 \mathrm{~g} / \mathrm{cm}^{3}, 62 \%, 26$ and $45 \%$ for Kasaoka clay, respectively. The sample is made by adding distilled water of which water content is about twice the liquid limit and stirred in the soil mixer. Akita peat is taken from peat layer spreading $2 \mathrm{~m}$ below from the ground surface in the suburb of Akita city, Japan. The sample is reconstituted to a paste, of which water content is about $800 \%$, by adding distilled water and stirring. Particle density of the sample is $1.64 \mathrm{~g} / \mathrm{cm}^{3}$, ignition loss is $76.5 \%$ and degree of decomposition is $75.5 \%$. These three samples were transferred under a vacuum to a preconsolidation cell and consolidated one dimensionally.

In order to confirm that the technique proposed in this paper does not depend on the testing system, two types of testing apparatus: an oedometer and a triaxial apparatus 
Table 1. Testing apparatuses, samples and test conditions

\begin{tabular}{c|l|c|c|c|c|c|c}
\hline $\begin{array}{c}\text { Testing } \\
\text { apparatus }\end{array}$ & Sample & $\begin{array}{c}\text { Consolidation } \\
\text { stress (kPa) }\end{array}$ & $\begin{array}{c}\text { frequency-swept } \\
\text { signal }\end{array}$ & $\begin{array}{c}\text { Initial height of } \\
\text { specimen }(\mathrm{mm})\end{array}$ & $\begin{array}{c}\text { Initial diameter of } \\
\text { specimen }(\mathrm{mm})\end{array}$ & $\begin{array}{c}\text { Dimensions of } \\
\text { transmitter BE (mm) }\end{array}$ & $\begin{array}{c}\text { Dimensions of } \\
\text { receiver BE (mm) }\end{array}$ \\
\hline \multirow{2}{*}{$\begin{array}{c}\text { Triaxial } \\
\text { apparatus }\end{array}$} & Kasaoka clay & 100 & LSSP & $100,75,50$ & 50 & $14.0 \times 10 \times 0.7$ & $3.0 \times 10 \times 0.7$ \\
\cline { 2 - 9 } & Akita peat & $80,100,200,300$ & TSP & 150 & 70 & $11.5 \times 10 \times 0.5$ & $11.1 \times 10 \times 0.5$ \\
\hline \multirow{2}{*}{ Oedometer } & NSF clay & $20,50,100$ & TSP & 75 & 60 & $7.4 \times 10 \times 0.5$ & $7.5 \times 10 \times 0.5$ \\
\cline { 2 - 9 } & Akita peat & $20,40,60,80$ & TSP & 75 & 60 & $7.4 \times 10 \times 0.5$ & $7.5 \times 10 \times 0.5$ \\
\hline
\end{tabular}

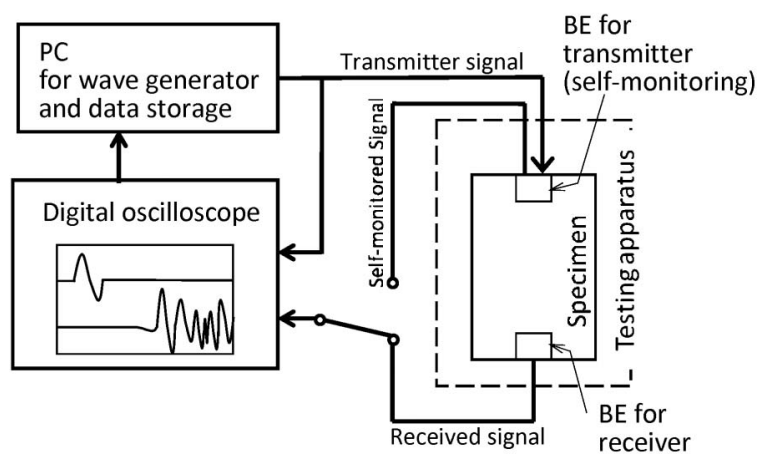

Fig. 2. Schematic view of bender element testing system

are prepared. These apparatuses are altogether different in lateral boundary conditions of specimen: rigid boundary can be assumed in oedometer, while flexible boundary in triaxial apparatus. Furthermore, two different dimensions of specimen and bender elements are examined on triaxial apparatus. A series of incremental loading consolidation tests are performed on these samples. In the tests using triaxial cell, specimens are isotropically consolidated in the triaxial cell. Note that the height of oedometer is modified to be available for specimen of 75 $\mathrm{mm}$ in height so that enough height could be ensured when highly compressible peat sample is consolidated. Testing apparatuses, samples and test conditions are summarized in Table 1.

At the end of the primary consolidation of each prescribed incremental stress, bender element tests were performed using two kinds of transmitter pulse: frequency-swept signal and one-cycle sine pulse of which frequency varies from 0.5 to $10 \mathrm{kHz}$ in incremental steps. As mentioned above, received wave of frequency-swept signal obtains frequency response of the system necessary for calculating received waves. Results of one-cycle sine pulse are compared with calculations. Figure 2 shows a schematic view of the testing system. Transmitter signal is generated by a Digital-Analog (DA) converter installed in the PC. Peak-to-peak voltage of transmitter signals is $\pm 10 \mathrm{~V}$. Both transmitted and received waves are monitored by a digital oscilloscope with sampling frequency of $100 \mathrm{kHz}$ or above and 4 times averaging. Electrode of the transmitter element is separated into two and can work as a self-monitoring element, of which output signal corresponds with the displacement of element itself.

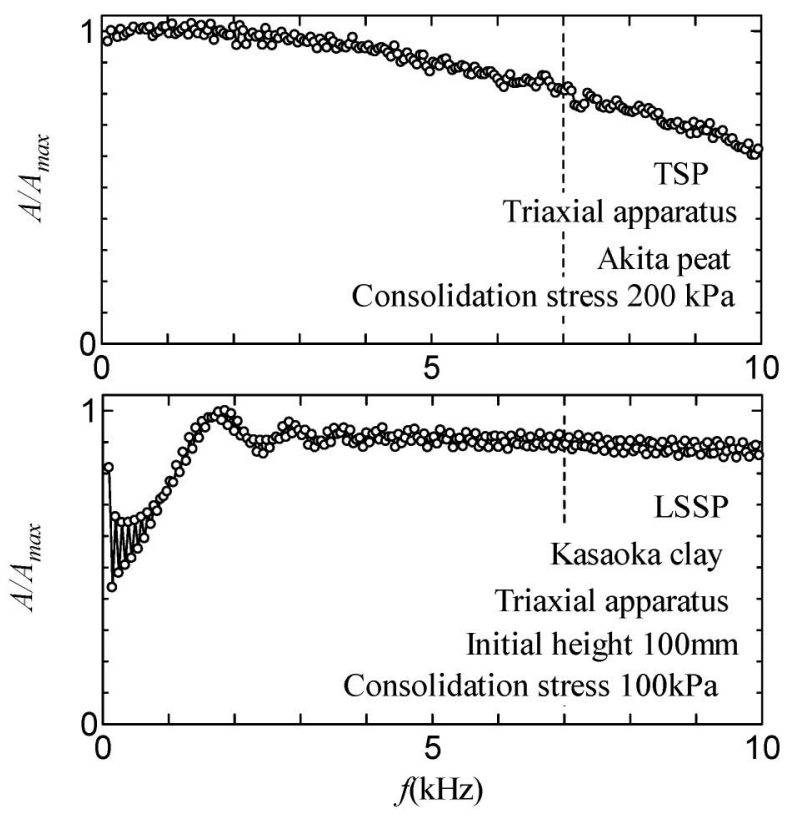

Fig. 3. Amplitude spectra of TSP and LSSP given by signals from self-monitoring element

\section{INPUT/OUTPUT CHARACTERISTICS OF THE TESTING SYSTEM}

Since the technique proposed in this paper is based on the linear system theory, it is important to confirm the linearity of testing system. As shown later (Fig. 7), as the system response is up to $7 \mathrm{kHz}$ for all tests, only frequencies under $10 \mathrm{kHz}$ is considered in this paper. Frequencyswept signals were designed to cover the range. The range is determined by parameters $N, m, f_{0}, \Delta f$ and $t_{\mathrm{t}}$ in Eqs. (4) and (5). These parameters should be determined in each case according to the range of system response so that the precise frequency response function can be identified. For example, if a harder sample such as sand is tested, frequency range would shift to the higher side.

Signals actually driving transmitter element are checked in Fig. 3, showing amplitude spectra of transmitted frequency-swept signals observed by monitor element. Output signal of self-monitoring element can represent the displacement of bender element itself (Nishio and Hotta, 2000). These spectra are slightly different from those in Fig. 1, which are based on Eqs. (4) and (5), and are not actual transmitted signals. Although the amplitude of the actual driving signal gradually decreases with 
frequency, especially in TSP, it is found that the amplitude is more than $80 \%$ of its maximum value at frequency of $7 \mathrm{kHz}$ and even $60 \%$ at $10 \mathrm{kHz}$.

Coherence function $R^{2}$ is used to represent the degree of linearity, as described in the following equation:

$$
R^{2}=\frac{\overline{\left|P_{\mathrm{xy}}\right|^{2}}}{\overline{P_{\mathrm{xx}}} \cdot \overline{P_{\mathrm{yy}}}}
$$

where $P_{\mathrm{xy}}$ is cross spectrum of received signal with trans-

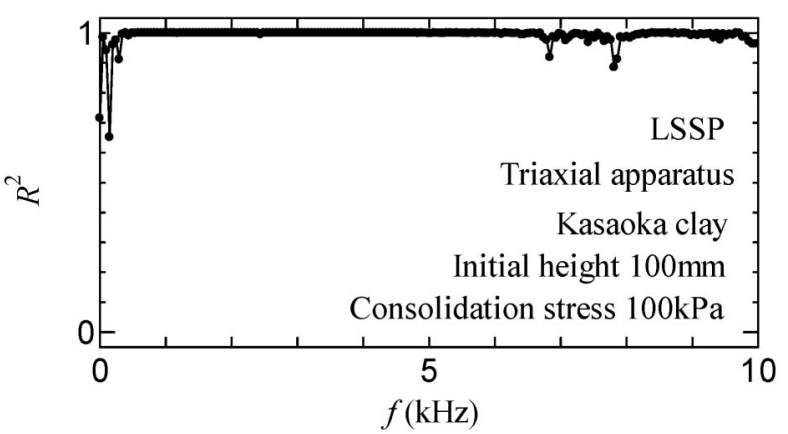

Fig. 4. Coherence for 4 time frequency-swept signal inputs

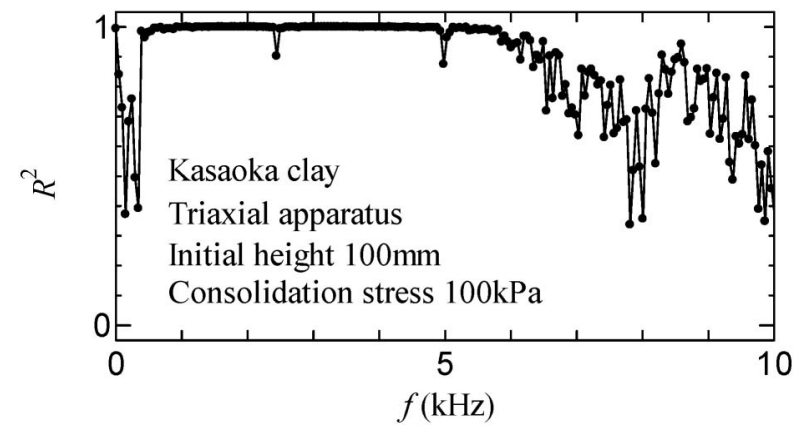

Fig. 5. Coherence for various signal inputs
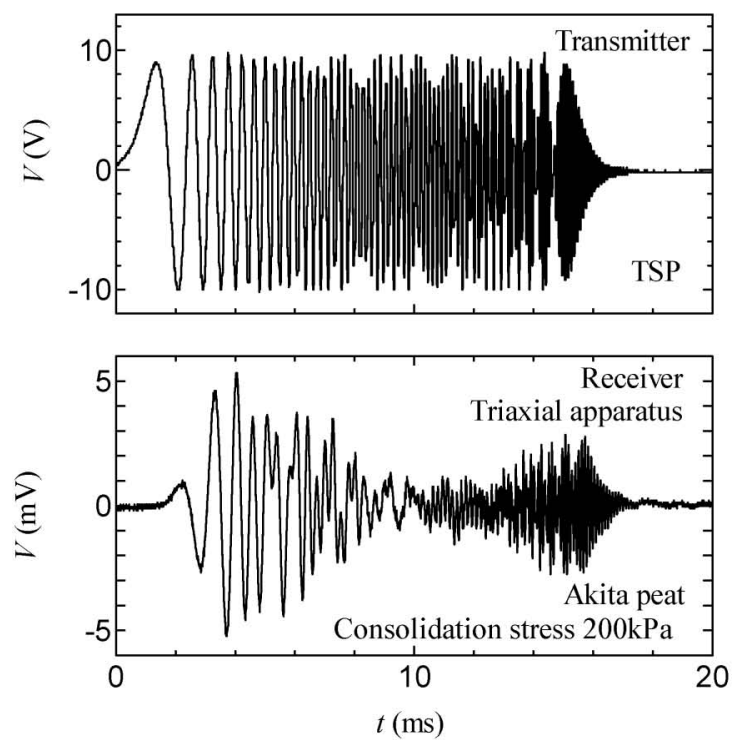

mitted signal, and $P_{\text {xx }}$ and $P_{\text {yy }}$ are mean amplitude of spectrum for transmitted and received signals, respectively. Figure 4 shows coherence function of received wave for LSSP. Since four types of signals are averaged in Fig. 4, coherence function in this figure represents the effect of random noise, which is a factor affecting the calculation accuracy. It can be seen that the value of coherence function is nearly 1 in the given frequency bandwidth. It follows that the system is less affected by random noise.

Coherence function of received signal for various transmitted wave, on the other hand, is shown in Fig. 5. Transmitted waves are LSSP and one-cycle sine pulses of which frequency are $0.5,1.0,2.0,3.0,4.0$ and $5.0 \mathrm{kHz}$. This figure is considered to represent the linearity of the system in a given frequency range. Coherence is approximately 0.8 or higher between 0.5 and $7 \mathrm{kHz}$ and indicates that the testing system is considered to show sufficiently linear behavior in this frequency range. Similar results are also shown for TSP.

\section{FREQUENCY RESPONSE AND IMPULSE RESPONSE OBTAINED FROM FREQUENCY-SWEPT SIGNAL}

Typical transmitted and received waveforms for TSP and LSSP under triaxial consolidation are shown in Fig. 6. Entire waveform of both transmitted and received waves must be captured to restore frequency response. The duration of transmitter signal can be varied by the parameters $m$ and $t_{\mathrm{t}}$ in Eqs. (4) and (5). The duration become long when these parameters are large. These parameters consequently affect the amplitude of frequency response because longer duration signal provides more energy. Therefore, when larger output is needed, longer signal should be used. However, signal of which length is longer than that of received wave decreases reso-
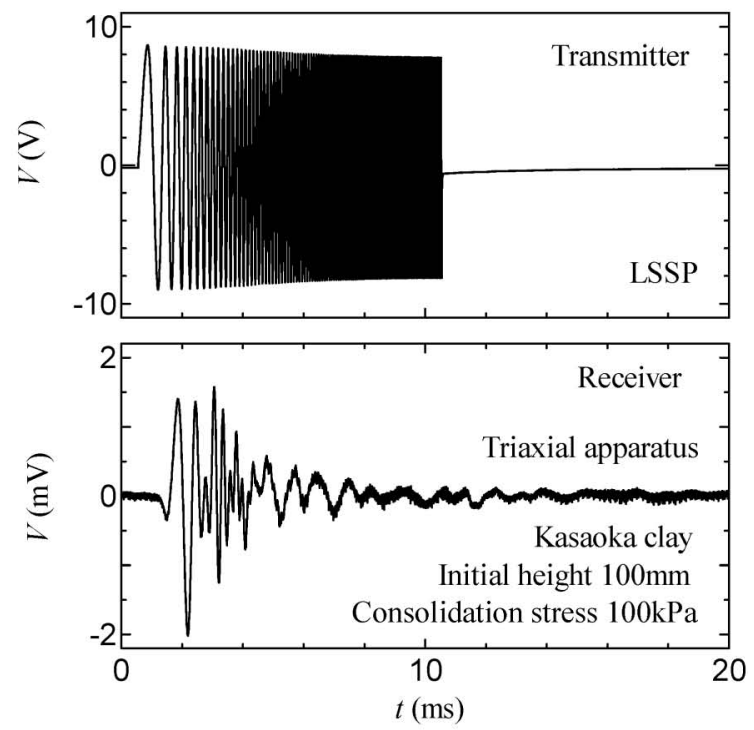

Fig. 6. Frequency-swept signals for transmitter and typical received waveforms 

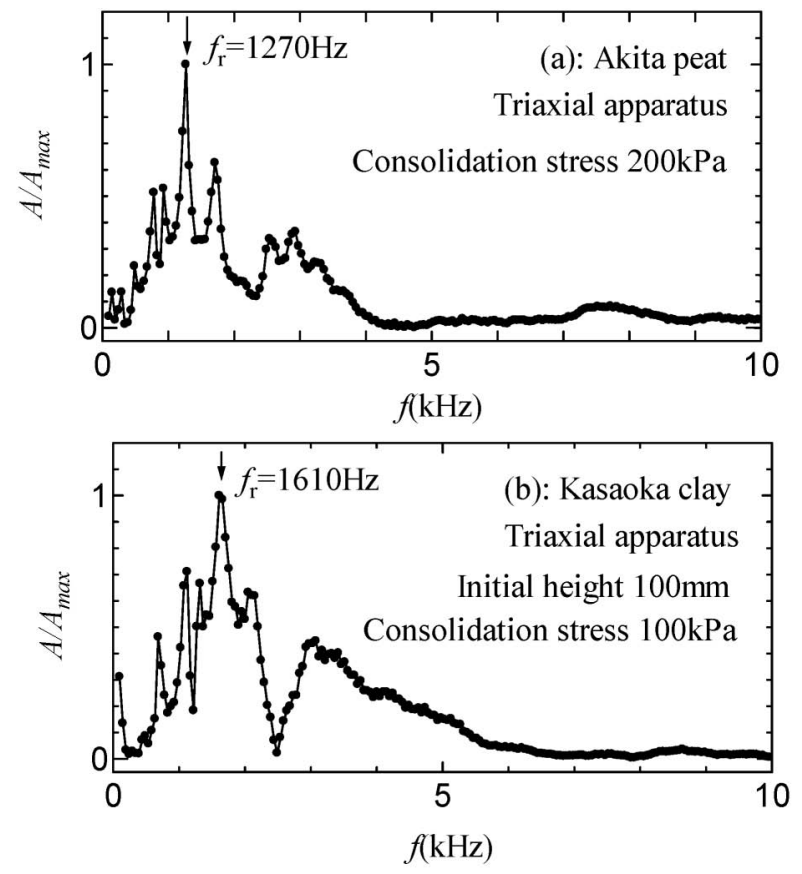

Fig. 7. Typical frequency responses restored by (a) TSP and (b) LSSP

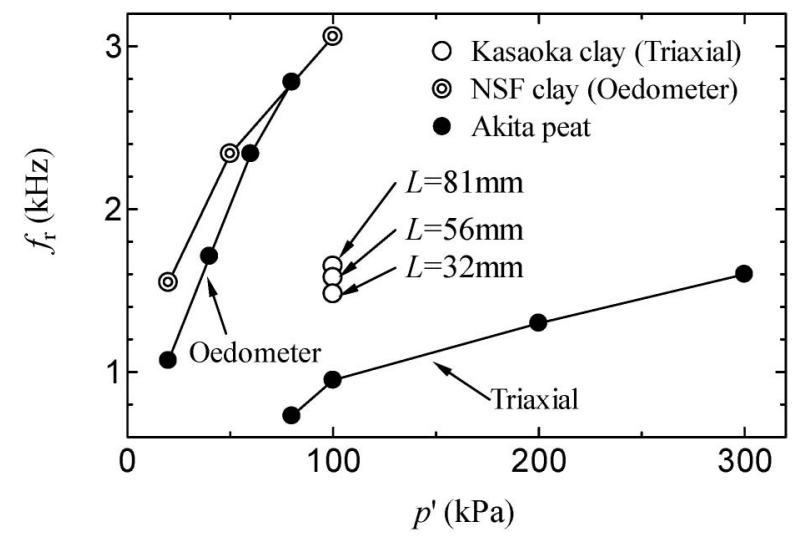

Fig. 8. Relationship of resonant frequency versus consolidation stress

lution of time scale for impulse response due to limitation of sampling number of digital oscilloscope or FFT analyzer. The decrease in resolution affects the accuracy of travel time determination. It follows that high resolution in time scale and high output are conflicting requirements. Hence parameters $m$ and $t_{\mathrm{t}}$ should be determined by balancing the resolution of output and time scale necessary for calculated wave.

Figure 7 shows frequency response obtained from received wave shown in Fig. 6. It is found that the range of frequency response in which amplitude exists is less than $7 \mathrm{kHz}$. There are differences in the shape of spectrum between these figures. The shape of spectrum is considered to be affected by the difference of testing system involving the boundary condition or dimensions of specimen, soil sample, consolidation stress and dimensions of bender elements. Resonant frequency, denoted as $f_{\mathrm{r}}$ in Fig. 7, can be an index which represents the characteris-
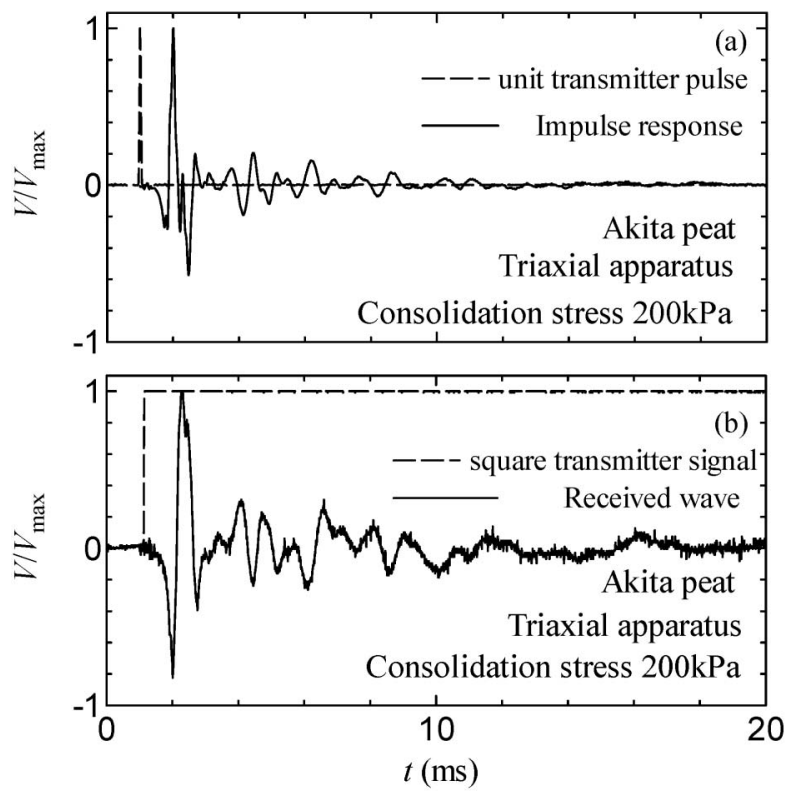

Fig. 9. Comparison between (a) impulse response and (b) received wave for square signal input

tics of the spectrum shape. Figure 8 shows change in resonant frequency with consolidation stress for each sample shown in Table 1. Results from triaxial apparatus are located below those from oedometer. This indicates that the boundary condition greatly affects resonant frequency. Resonant frequencies are also affected by consolidation stress and type of soil sample. It can also be seen from the results by Kasaoka clay that resonant frequency is little affected by tip-to-tip distance $L$. Since resonant frequency is mainly affected by soil density and stiffness (see also Blewett et al., 2000; Lee and Santamarina, 2005), this difference or change in resonant frequency can reasonably be explained by these soil characteristics: Resonant frequency increases with consolidation stress because both soil density and stiffness increase with consolidation stress. Resonant frequency for Akita peat also locates below the data for clay samples due to its low density and stiffness, if the boundary conditions are the same.

As they are a pair of Fourier transform (see also in Eqs. (2) and (3)), impulse response can be given by calculating inverse FFT of frequency response. The physical meaning of impulse response is a received wave for ideal unit transmitter pulse which is given by a delta function. Therefore impulse response should be, in principle, similar to the received wave for impulse-type transmitter signal such as square signal. Figure 9(a) shows an impulse response for Akita peat calculated from the frequency response function shown in Fig. 7(a) and a unit transmitter pulse. Figure 9(b) shows a square transmitter signal and corresponding received waveform. Indeed, it seems that waveform of impulse response is similar to received waveform shown in Fig. 9(b). This agreement indicates that the impulse response enables to estimate the travel time of shear wave as it is (Ogino et al., 2006). 


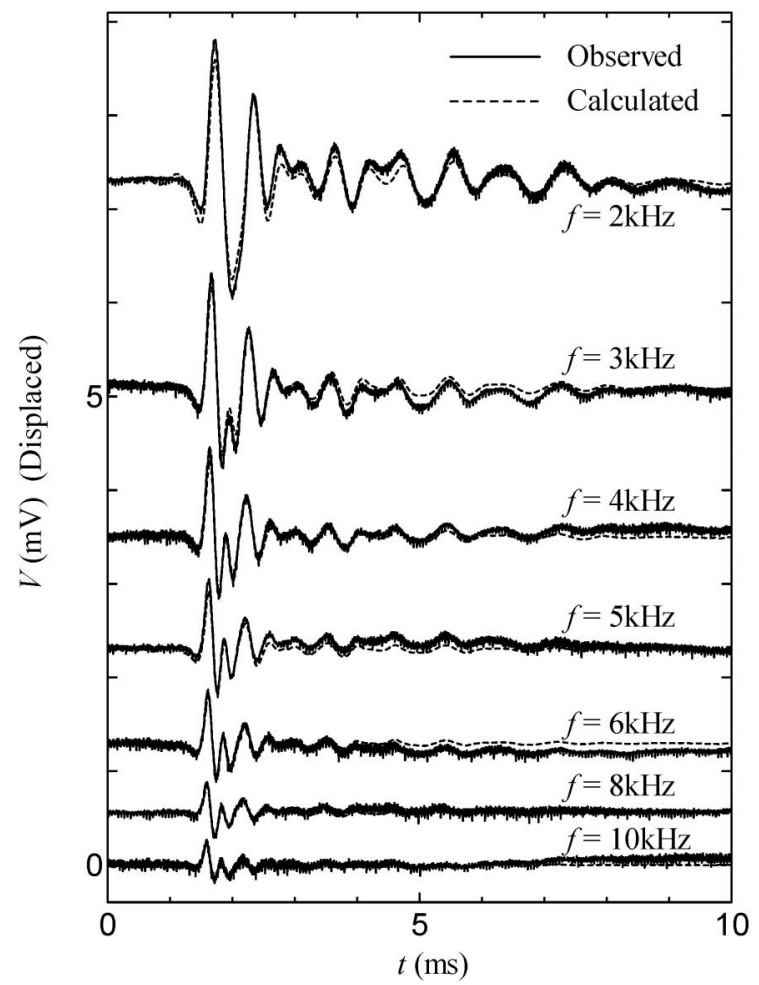

Fig. 10. Comparison between observed and calculated received waves for various frequency sine inputs (Kasaoka clay, triaxial apparatus, consolidation stress: $100 \mathrm{kPa}$, Initial height: $100 \mathrm{~mm}$ )

\section{COMPARISON BETWEEN RECONSTRUCTED AND OBSERVED WAVEFORM}

Seven cases of calculated and observed received waveforms for one-period sine transmitter signal are compared in Fig. 10. Frequencies of one-period sine transmitter signal are 2, 3, 4, 5, 6, 8, $10 \mathrm{kHz}$. Calculated waves are given by Eq. (3) in the frequency domain. In these cases, $X(f)$ should be FFT of each one-period sine transmitter signal and $Z(f)$ should be the frequency response function given by the cross-spectrum of frequency-swept signal and corresponding received signal shown in the right side of Fig. 6. Hence, calculated received waves in time domain are given by the inverse FFT of Eq. (3). It can be seen that there is little difference between calculation and observation through transmitting frequency between 2 and $10 \mathrm{kHz}$. Amplitude of calculated wave corresponds with that of observed wave in each case. Decreasing of amplitude by increasing frequency is also expressed. Moreover, distortion resulted from near-field effect is described in calculated waveforms: clear troughs are found in the beginning of both observed and calculated waveforms when transmitting frequency is less than $5 \mathrm{kHz}$. It follows that this technique can restore any received waves for arbitrary transmitter signal. This agreement is also shown in some characteristic points commonly used as travel time determination. Calculated and observed waveforms are again compared in Fig. 11. Figure 11(a) is the result for Akita peat by triaxial apparatus and Fig. 11(b) is for NSF clay by oedometer. Time axis is enlarged so that the difference of these points can be clear. Some characteristic points on
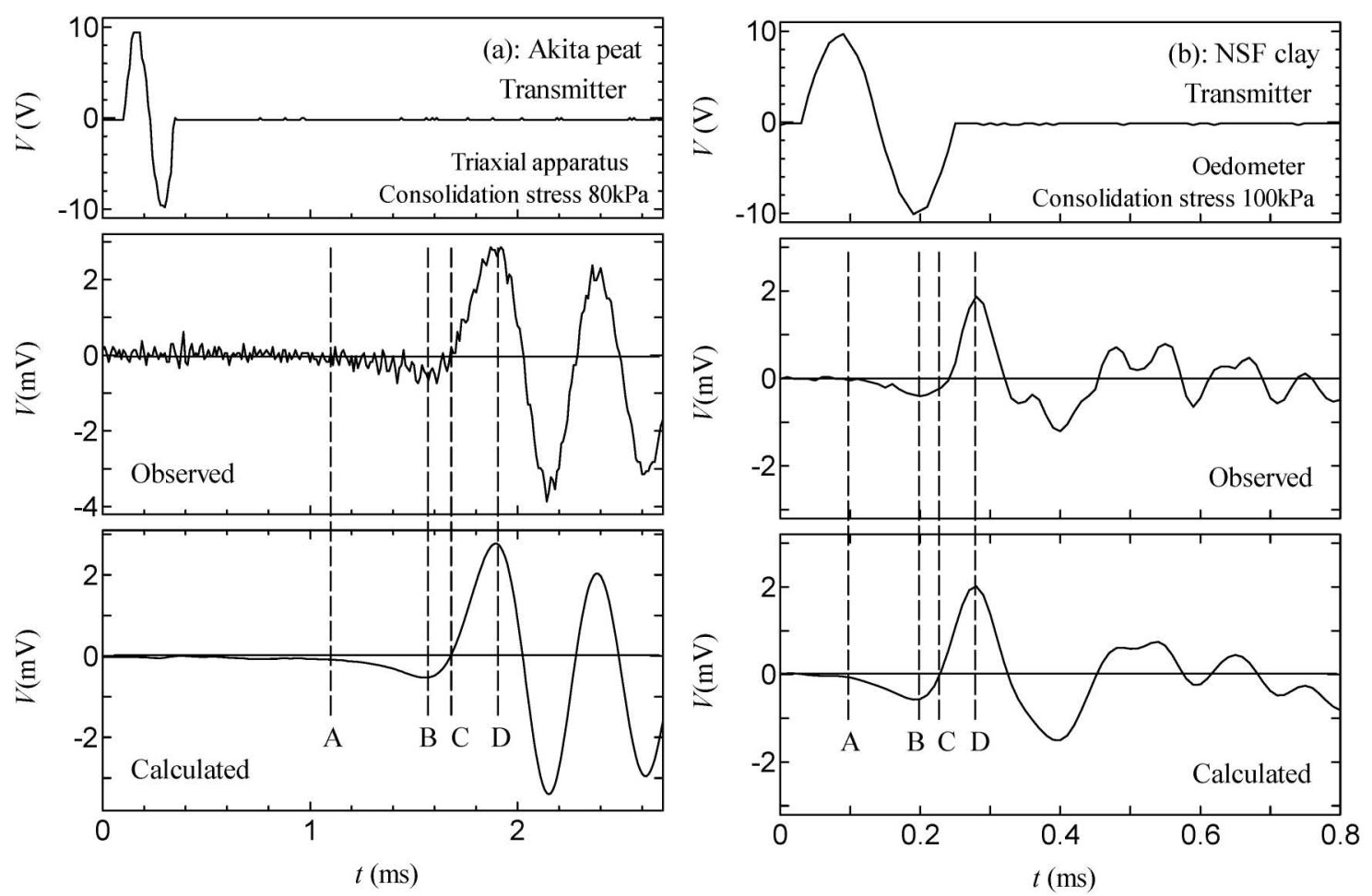

Fig. 11. Comparison of arrival time at characteristic points (a) Akita peat and (b) NSF clay 
calculated waveforms are also denoted as point A (first deflection), point B (first bump of reverse), point C (zerocrossing point) and point D (first peak) in Fig. 11. Although slight difference is found at the point $\mathrm{C}$ for NSF clay (Fig. 11(b)), there is little difference in arrival times determined at each of these characteristic points between calculation and observation. This means that this technique can provide shear wave velocity which is equivalent to that obtained from observed wave in time domain technique regardless of where the arrival point is of the shear wave. Consequently, shear wave velocity can be verified for any transmitting waveforms even after the bender element test is completed. This may be adopted, for example, when reasonable shear wave velocity is not obtained from ordinary bender element test because of uncertainty of travel time due to influence of near-field effect. Furthermore, it should be noted that testing system and frequency-swept signal used to identify the frequency response are different among in Figs. 10, 11(a) and 11(b): soil samples, testing apparatus, consolidation stress, dimensions of bender elements and type of frequency-swept signals are all different in the results depicted in these figures. It follows that this technique can be applied irrespective of sample, testing apparatus and type of frequency-swept signal.

There is an advantage in signal/noise ratio for calculated wave. Disturbance due to random noise coming from outside of the testing system can be seen in the observed received waves shown in Figs. 10 and 11(a), while smooth lines are shown in the calculated waves. This contrast becomes more significant when frequency of transmitted signal increases because amplitude of observed wave becomes smaller while amplitude of noise remains. As mentioned above, since amplitude of frequency-swept signal has high energy because of its long duration, this high energy makes improvements in signal/noise ratio of received wave for frequency-swept signal and eventually calculated wave. In fact, there is little disturbance due to random noise in the received wave shown in Fig. 6 or restored impulse response shown in Fig. 9. This is the reason why calculated wave does not contain random noise. This can be advantageous when sufficient amplitude of received wave cannot be ensured, for example, because of long distance between transmitter and receiver elements or high-frequency of transmitting signal.

\section{SHEAR WAVE VELOCITY OBTAINED FROM CALCULATED WAVE}

Shear wave velocities obtained from observed and calculated waveforms are compared in Fig. 12. As mentioned above, although it remains unclear which point from A to D in Fig. 11 is the reasonable arrival point, shear wave velocities are temporarily calculated from travel time which is determined by the time between the start point of transmitting wave and zero-crossing point of received wave (point $\mathrm{C}$ in Fig. 11). Most of data plots are on the one-to-one relation and spread within the range from $+10 \%$ to $-10 \%$. The difference is less than

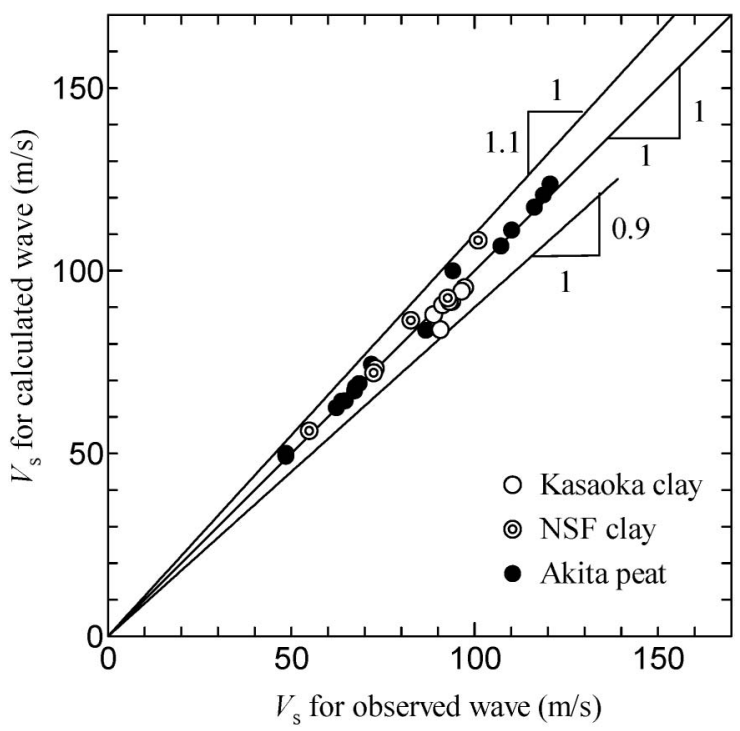

Fig. 12. Comparison of shear wave velocities between observed and calculated waves

$8 \%$. This result confirms that calculated wave can be an alternative to observed wave to estimate the shear wave velocity regardless of soil samples and testing apparatuses.

\section{CONCLUSION}

A technique for identifying frequency response of bender element testing system based on linear system theory is shown. Frequency responses for several different testing systems involving conditions of specimen and dimensions of bender elements are experimentally identified using frequency-swept signal and applied to compute the received waveform for arbitrary transmitted wave. Calculated waveforms are compared with experimental data. The main observations from this study are as follows:

1. Received waveforms of bender element test could be reconstructed by frequency response of the testing system obtained by frequency-swept signals. Two types of frequency-swept signals designed by different formula were tested. Calculated waveforms agreed with observed ones irrespective of the type of frequencyswept signal or frequency.

2. This method for received wave reconstruction could be applied irrespective of testing system. Test results which examined the influence of testing system using different samples, different testing apparatus and different dimensions of specimen and bender elements did not affect the applicability of this technique.

3. The shape of frequency response changed depending on testing system. In particular, change in resonant frequency was reasonably explained by change of soil stiffness and density.

4. Shear wave velocities obtained from calculated and observed waves showed good agreement in any testing system. Difference of shear wave velocities was less than $8 \%$. 


\section{ACKNOWLEDGMENTS}

This research was partially supported by the Ministry of Education, Science, Sports and Culture, Grant-in-Aid for Young Scientists (B), 19760322, 2007.

\section{REFERENCES}

1) Aoshima, N. (1981): Computer-generated pulse signal applied for sound measurement, Journal of the Acoustical Society of America, 69, 1484-1488.

2) Arulnathan, R., Boulanger, R.W. and Riemer, M. F. (1998): Analysis of bender element tests, Geotechnical Testing Journal, 21(2), 120-131.

3) Blewett, J., Blewett, I. J. and Woodward, P. K. (2000): Phase and amplitude responses associated with the measurement of shearwave velocity in sand by bender elements, Canadian Geotechnical Journal, 37, 1348-1357.

4) Brignoli, E. G. M., Gotti, M. and Stokoe, K. H., II (1996): Measurement of shear waves in laboratory specimens by means of piezoelectric transducers, Geotechnical Testing Journal, 19(4), 384-397.

5) Dyvik, R. and Madshus, C. (1985): Lab measurements of $G_{\max }$ using bender elements, Proc. ASCE Annual Convention: Advances in the Art of Testing Soils under Cyclic Loading, Detroit, MI, 186-196.

6) Dyvik, R. and Olsen, T. S. (1991): $G_{\max }$ measured in oedometer and
DSS tests using bender elements, Norges Geotekniske Institutt, 181, $1-4$.

7) Greening, P. D. and Nash, D. F. T. (2004): Frequency domain determination of $G_{0}$ using bender elements, Geotechnical Testing Journal, 27(3), 288-293.

8) Jovicic, V., Coop, M. R. and Simic, M. (1996): Objective criteria for determining $G_{\max }$ from bender element tests, Geotechnique, 46(2), 357-362.

9) Lee, J. S. and Santamarina, J. C. (2005): Bender elements: performance and signal interpretation, Journal of Geotechnical and Geoenvironmental Engineering, ASCE, 131(9), 1063-1070.

10) Nishio, S. and Hotta, H. (2000): Applicability of self-monitoring bender element, Proc. Japanese Geotechnical Society, 261-262 (in Japanese).

11) Ogino, T., Oikawa, H., Mitachi, T., Tsushima, M. and Nishida, K. (2006): Shear wave velocity by TSP applied bender element test in sand, Journal of JSCE, 62(1), 169-174 (in Japanese).

12) Sanchez-Salinero, I., Roesset, J. M. and Stokoe, K. H. (1986): Analytical study of body wave propagation and attenuation, Geotechnical Engineering Report No GR86-15, Civil Engineering Department, University of Texas at Austin.

13) Santamarina, J. C. and Fam, M. A. (1997): Interpretation of bender element tests (discussion), Geotechnique, 47(4), 873-877.

14) Shirley, D. J. and Hampton, L. D. (1978): Shear-wave measurements in laboratory sediments, Journal of the Acoustical Society of America, 63(2), 607-613.

15) Viggiani, G. and Atkinson, J. H. (1995): Interpretation of bender element tests, Geotechnique, 45(1), 149-154. 